\title{
Tracing of Skew Rays
}

\section{By Donald P. Feder}

\begin{abstract}
This paper presents a set of convenient recurrence formulas for the tracing of skew rays by means of a desk calculator. Because of the increased speed and field coverage of modern lenses, it is frequently desirable to trace such rays in making a complete study of an optical system.

The formulas apply to systems of spherical refracting surfaces. The system need not be centered.

Particularly convenient check formulas are available, which need be applied only at the final surface.
\end{abstract}

A complete study of an optical system appears to demand some knowledge of the behavior of the general or skew rays. A computational process for obtaining this information by tracing such rays, which has proved convenient in practice, will now be described.

It is contemplated that these formulas will be used with a desk calculating machine. When sucb a calculator is used, twelve numbers per surface must be written down for each ray.

An advantage of the method is the existence of a simple check that need be applied only at the final surface.

Brief derivation. We consider a system of spherical refracting surfaces. The system need not be centered.

We denote the incident and refracted rays by the unit vectors $\boldsymbol{Q}$ and $\boldsymbol{Q}^{\prime}$, and the radius vector to the center of curvature from the point of incidence by $\boldsymbol{R}$. Bold-faced type will be used to denote vectors.

We define a new vector $\boldsymbol{P}$

$$
\boldsymbol{P}=N \quad \boldsymbol{Q} \times \boldsymbol{R} .
$$

The law of refraction becomes

$$
\boldsymbol{P}=N^{\prime} \quad \boldsymbol{Q}^{\prime} \times \boldsymbol{R},
$$

where $N$ and $N^{\prime}$ are the refractive indices of the surrounding media.

We can now choose our independent variables from the set of rectors $(\boldsymbol{P}, \boldsymbol{Q}, \boldsymbol{R})$. Any two of these determine the direction and location of the ray and may be used as independent variables. An obvious choice is the pair $\boldsymbol{Q}$ and $\boldsymbol{R}$, but $\boldsymbol{P}$ and $\boldsymbol{Q}$ yield simpler equations, particularly a simpler transfer.

We eliminate $\boldsymbol{R}$ from eq 1 and 2 in order to obtain $\boldsymbol{Q}^{\prime}$ as a function of $\boldsymbol{P}$ and $\boldsymbol{Q}$. Multiplying eq 1 vectorially by $\boldsymbol{Q}$, one can obtain $\boldsymbol{R}$, which is then substituted in eq 2. After solving the resulting equation for $\boldsymbol{Q}^{\prime}$, we obtain

$$
\boldsymbol{Q}^{\prime}=a \boldsymbol{Q}+b \boldsymbol{P} \times \boldsymbol{Q},
$$

where

$$
\begin{aligned}
& a=\frac{\boldsymbol{P}^{2}+N N^{\prime}\left(\boldsymbol{Q}^{\prime} \cdot \boldsymbol{R}\right)(\boldsymbol{Q} \cdot \boldsymbol{R})}{N N^{\prime} \boldsymbol{R}^{2}}, \\
& b=\frac{N^{\prime}\left(\boldsymbol{Q}^{\prime} \cdot \boldsymbol{R}\right)-N(\boldsymbol{Q} \cdot \boldsymbol{R})}{N N^{\prime} \boldsymbol{R}^{2}} .
\end{aligned}
$$

$(\boldsymbol{Q} \cdot \boldsymbol{R})$ and $\left(\boldsymbol{Q}^{\prime} \cdot \boldsymbol{R}\right)$ may be evaluated by squaring eq 1 and 2 respectively. We find

$$
\begin{gathered}
N^{2}(\boldsymbol{Q} \cdot \boldsymbol{R})^{2}=N^{2} \boldsymbol{R}^{2}-\boldsymbol{P}^{2} \\
{N^{\prime \prime}}^{\prime 2}\left(\boldsymbol{Q}^{\prime} \cdot \boldsymbol{R}\right)^{2}=N^{\prime 2} \boldsymbol{R}^{2}-\boldsymbol{P}^{2}
\end{gathered}
$$

Given $\boldsymbol{P}$ and $\boldsymbol{Q}$, these equations enable us to find $\boldsymbol{Q}^{\prime}=\boldsymbol{Q}_{1}$. It remains only to find $\boldsymbol{P}_{1}$ for the following surface in terms of $\boldsymbol{P}$ and $\boldsymbol{Q}$ at the preceding one.

In figure $1 O$ and $O_{1}$ are the respective centers, and $A$ and $A_{1}$ are the points of incidence of the ray. In general the quadrilateral is not plane. 


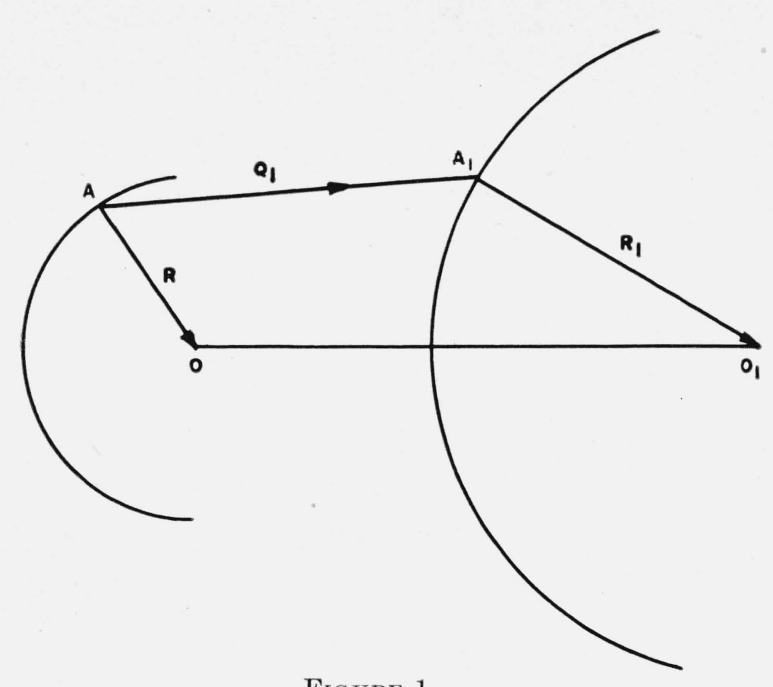

FiguRE 1.

$\boldsymbol{R}, \boldsymbol{Q}_{1}$, and $\boldsymbol{R}_{1}$ denote vectors.

Denoting the vector from $O$ to $O_{1}$ by $\boldsymbol{T}$, one has immediately

$$
\boldsymbol{R}_{1}+\overline{A A}_{1} \boldsymbol{Q}_{1}=\boldsymbol{R}+\boldsymbol{T}
$$

Multiplying vectorially by $N^{\prime} \boldsymbol{Q}^{\prime}=N_{1} \boldsymbol{Q}_{1}$, the term in $\overline{A A}_{1}$ vanishes, and we have

$$
\boldsymbol{P}_{1}=\boldsymbol{P}+\boldsymbol{Q}_{1} \times \boldsymbol{t}
$$

where $\boldsymbol{t}=N_{1} \boldsymbol{T}$

Summary in scalar form. Since the vast majority of optical systems are centered, let us suppose that $\overline{O O}_{1}$ lies on the optical axis. We may take the $x$ axis along this line so that $t_{y}=t_{z}=0$.

Suppose that $\boldsymbol{Q}=(X, Y, Z)$ and $\boldsymbol{P}=\left(P_{x}, P_{y}, P_{z}\right)$ are given in scalar form. Defining the auxiliary constants $r=N|\boldsymbol{R}|$ and $r^{\prime}=N^{\prime}|\boldsymbol{R}|$, and writing

$$
\begin{gathered}
P^{2}=P_{x}^{2}+P_{y}^{2}+P_{z}^{2} \\
g^{2}=r^{2}-P^{2} \\
g^{\prime 2}=r^{\prime 2}-P^{2} \\
a=\frac{g^{\prime} g+P^{2}}{r r^{\prime}} \\
b=\frac{g^{\prime}-g}{r r^{\prime}}
\end{gathered}
$$

$$
\left.\begin{array}{l}
X_{1}=a X+b\left[P_{y} Z-P_{z} Y\right] \\
Y_{1}=a Y+b\left[P_{z} X-P_{x} Z\right] \\
Z_{1}=a Z+b\left[P_{x} Y-P_{y} X\right]
\end{array}\right\}
$$

$$
\left.\begin{array}{l}
P_{1 x}=P_{x} \\
P_{1 y}=P_{y}+t_{x} Z_{1} \\
P_{1 z}=P_{z}-t_{x} Y_{1}
\end{array}\right\}
$$

The signs of $g$ and $g^{\prime}$ are identical and are positive for surfaces convex toward the entering rays.

Equations 10 through 16 inclusive, form the complete set for systems of spherical surfaces.

One notices that $P_{x}$ is an invariant of the ray. $P_{x}=0$ implies that the ray lies in a meridian plane.

Plane surfaces. At a plane refracting surface normal to the optical axis, the refraction equations are greatly simplified.

From eq 1 and 2 by taking $\boldsymbol{R}=\boldsymbol{i}$ one obtains

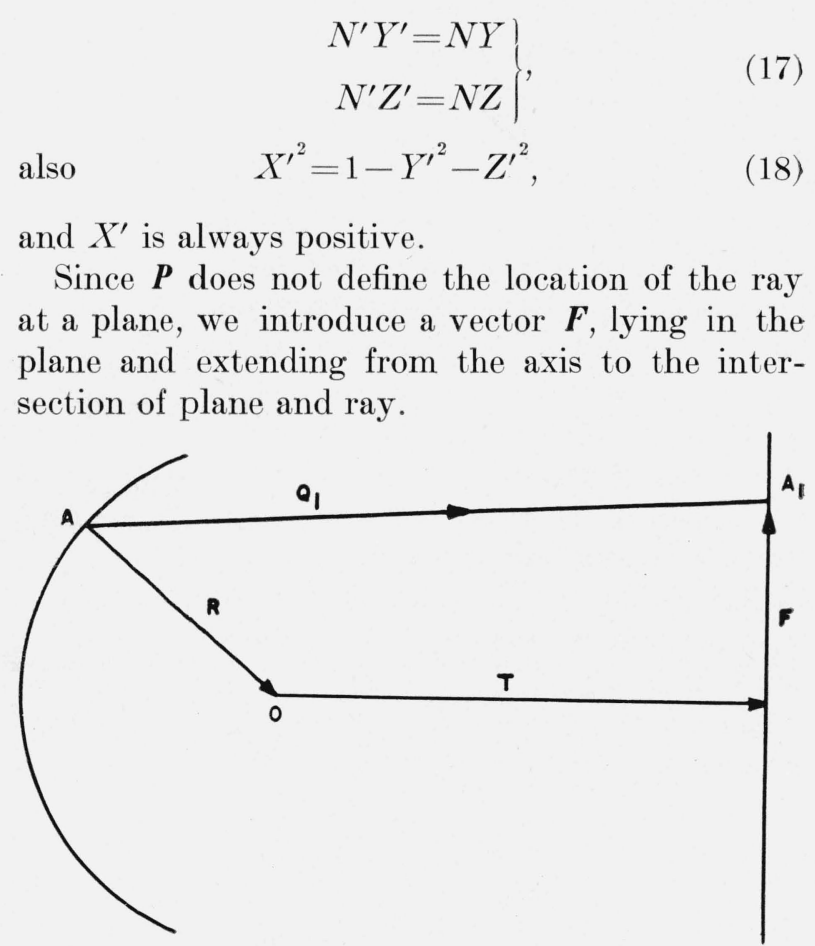

Figure 2.

$\boldsymbol{R}, \boldsymbol{Q}_{1}, \boldsymbol{F}$, and $\boldsymbol{T}$ denote vectors.

From figure 2 we obtain $\overline{A A}_{1} \boldsymbol{Q}_{1}=\boldsymbol{R}+\boldsymbol{T}+\boldsymbol{F}$ and multiplying by $N_{1} \boldsymbol{Q}_{1}$ as before, we have

$$
\boldsymbol{P}=N_{1} \boldsymbol{F} \times \boldsymbol{Q}_{1}+\boldsymbol{t} \times \boldsymbol{Q}_{1}
$$

where $\boldsymbol{t}=N_{1} \boldsymbol{T}$. In scalar form, if we take $t_{y}=t_{z}=0$

$$
\left.\begin{array}{l}
P_{x}=N_{1}\left[F_{y} Z-F_{z} Y_{1}\right] \\
P_{y}=N_{1} F_{z} X_{1}-t_{x} Z_{1} \\
P_{z}=-N_{1} F_{y} X_{1}+t_{x} Y_{1}
\end{array}\right\}
$$


Evidently eq 20 may be used to obtain $\boldsymbol{F}$ in terms of $\boldsymbol{P}$ or conversely. Frequently they are used as initial and final equations as well as at refracting surfaces.

Checking equations. At each surface

$$
\left.\begin{array}{l}
\boldsymbol{P} \cdot \boldsymbol{Q}=\boldsymbol{P} \cdot \boldsymbol{Q}_{1}=0 \\
\boldsymbol{Q}^{2}=\boldsymbol{Q}_{1}^{2}=1
\end{array}\right\},
$$

from eq 1 or 2 . It is important to note that these checks need be applied only at the final surface.

For we have from eq 3 by scalar multiplication

$$
\boldsymbol{P} \cdot \boldsymbol{Q}^{\prime}=a \boldsymbol{P} \cdot \boldsymbol{Q}
$$

and similarly from eq 9

$$
\boldsymbol{P}_{1} \cdot \boldsymbol{Q}_{1}=\boldsymbol{P} \cdot \boldsymbol{Q}_{1}=a \boldsymbol{P} \cdot \boldsymbol{Q} .
$$

Furthermore, since $a$ can be shown to be always nonzero, it follows that if $\boldsymbol{P} \cdot \boldsymbol{Q}$ is nonzero at any point, it remains nonzero. This implies that an error that causes $\boldsymbol{P} \cdot \boldsymbol{Q}$ to differ from zero at any surface will appear at the final surface.

Similarly, if any error is made so that $\boldsymbol{Q}^{2}-1 \not \equiv 0$, the error will appear at the final surface.

In addition one must have at each surface

$$
\left.\begin{array}{l}
a^{2}+b^{2} \boldsymbol{P}^{2}-1=0 \\
P^{2}-\boldsymbol{P}_{x}^{2}-P_{y}^{2}-P_{z}^{2}=0
\end{array}\right\} .
$$

These checks should guarantee the accuracy of the final data, provided no errors are made in the constants.

Using this method, the accuracy of the results is not limited by a particular set of tables. All that is needed is two square roots, and this may be obtained, with any desired accuracy, by the usual iteration formulas. It is very easy to obtain results to 10 significant figures by employing at most a small table.

Washington, March 30, 1950. 